\title{
HYDRODYNAMICAL SIMULATIONS AS PROBES FOR THE STRUCTURE OF THE GALACTIC CENTER
}

the central region of $N G C 4321$

\author{
K. WADA, T. MINEZAKI \\ National Astronomical Observatory, Mitaka, Tokyo 181, Japan \\ K. SAKAMOTO \\ Caltech, Radio Astronomy Department, 105-24, Pasadena, CA \\ 91125
}

AND

H. FUKUDA

Department of Physics, Hokkaido University, Sapporo 060, Japan

Numerical modeling of the interstellar gas in galaxies is an effective approach to infer galactic gravitational structure. This is because the dynamical behavior of gas is very sensitive to the background gravitational potential. Since the dynamical resonances depend closely on the mass distribution and the pattern speed of the non-axisymmetric component, it is possible to determine these dynamical parameters by comparison of numerical simulations and gas observations.

We have compared the high resolution $\mathrm{CO}$ interferometry observations (Sakamoto et al. 1995) with the two-dimensional hydrodynamical simulations of gas disks in gravitational potentials that suitably match the nuclear region of NGC 4321. For constructing the gravitational potential, we have used both a $K^{\prime}$ band image with a constant $M / L_{K}$ and an analytical description of a bar, in which $M / L_{K}$ is not constant. The hydrodynamical simulations were performed using the SPH method taking into account the self-gravity of the gas. Assuming that the nuclear bar $(R \sim 16 \operatorname{arcsec}$ $\sim 1.3 \mathrm{kpc}$ ) observed in the near-infrared contributes mainly to the gaseous dynamics in the nuclear bar region, the following points are revealed:

1. The best set of analytical bar model parameters for reproduction of the observations was determined. The best pattern speed of the inner bar is $65 \mathrm{~km} \mathrm{~s}^{-1} \mathrm{kpc}^{-1}$, which is consistent with the value estimated by Knapen et al. (1995b), but is about three times larger than that of the outer bar suggested by Sempere et al. (1995). 
2. The total mass of gas in the region of $R<1.5 \mathrm{kpc}$ was estimated in a dynamical way to be $\sim 1.0-1.3 \times 10^{9}\left(T_{g} / 10^{4} \mathrm{~K}\right)^{1 / 2} \mathrm{M}_{\odot}$, where $T_{g}$ is the gas kinematic temperature. This estimate is independent of that from CO luminosity, but agrees with it within a factor of 2 .

3. The observed morphology of the molecular gas is a short-lived and recurrent structure. The spiral structure lasts for about $10^{7} \mathrm{yr}$ at the observed position, and it is produced again after about $10^{8} \mathrm{yr}$.

4. The models with a constant mass-to-luminosity ratio $\left(M / L_{K}\right)$ and a single pattern speed do not reproduce the observations.

5. A two-dimensional map of $M / L_{K}$ was derived from the potential of the best fit model and the $K^{\prime}$ band image (Fig. 1). The $M / L_{K}$ ranges from 0.2 to $1.2(M / L)_{\odot}$ at $R<1.5 \mathrm{kpc}$. The $M / L_{K}$ is not constant but strongly affected by star formation. Thus the $K^{\prime}$ luminosity is not an accurate tracer of mass distribution in the central region of NGC 4321.

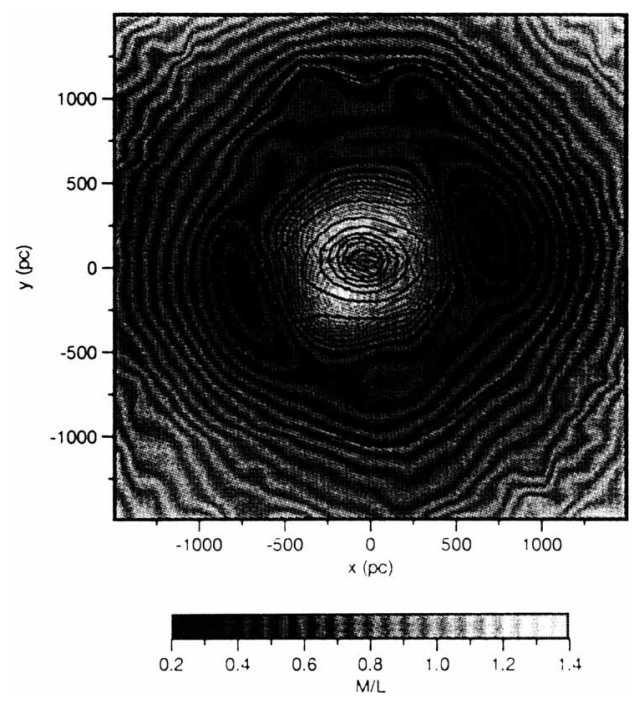

Figure 1. Mass-to-luminosity ratio $\left(M / L_{K}\right)$ map in the face-on view derived the mass distribution for the best fit analytical bar model with an extended disk potential and $K^{\prime}$ band image (contours, see Sakamoto et al. 1995). The color represents $M / L_{K}$ in the unit of $(M / L)_{\odot}$.

\section{References}

Knapen J. H., Beckman J. E., Heller C. H., Shlosman I., de Jong R. S., 1995b, ApJ, 454. 623

Sakamoto K., Okumura S., Minezaki T., Kobayash Y., Wada K. 1995, AJ, 110, 2075

Sempere M. J., Garcia-Burillo S., Combes F., Knapen J. H. 1995, A\&Ap, 296, 45 